\title{
Reciclaje de PET: Evaluación de la eficiencia de separación del contaminante PVC
}

\section{PET recycling: Evaluation of the efficiency of contaminant separation PVC}

\author{
Anie Karina da Rosa Oliveira, Carlos Henrique Michelin Beraldo y Ruth Marlene Campomanes Santana \\ Universidade Federal do Rio Grande do Sul. Av. Bento Gonçalves 9500, Agronomia, Porto Alegre, Brasil
}

\section{RESUMEN}

Uno de los plásticos convencionales más utilizados por la sociedad es el PET (polietileno tereftalato), ampliamente utilizado en la producción de botellas para bebidas carbonatadas y de agua mineral. Otro plástico que también es utilizado para esa aplicación, pero en menor cantidad es el PVC, (policloruro de vinilo). El reciclaje de polímeros es importante y ventajosa pero existen también desventajas como el gran volumen de residuo generado y el agotamiento de los rellenos sanitarios, entre otros. Existen diversas técnicas de separación de materiales poliméricos, siendo el más usado, la separación por diferencia de densidades (gravedad) pues es uno de los métodos más fáciles y barato de ser aplicado; el gran problema de ese método es la separación de materiales con densidades semejantes, como es el caso del PET y PVC, entre 1,30-1,37 $\mathrm{g} / \mathrm{cm}^{3}$ imposibilitando la separación de los mismos por esa técnica. El gran problema es la contaminación de PVC en el PET, pues ocasiona manchas en el PET transparente así como su degradación. En este sentido, el objetivo de este estudio es determinar una metodología más eficiente y viable económicamente de separación de PVC en PET a través del método de flotación. El primer paso fue tratar las muestras con solución alcalina de $\mathrm{NaOH}$ en temperaturas de $25^{\circ} \mathrm{C}$ y $80^{\circ} \mathrm{C}$ (tratatamento termoquímico), con el objetivo de modificar la superfície del PET, y con esto aumentar a su energia superfícial (ES). Para la determinación de la ES fueron realizadas las medidas de ángulo de contacto usando un goniómetro y en el ensayo de flotación fueron utilizadas soluciones de tensoativo MIBC y en diferentes pHs. Resultados de las condiciones de tratamiento termoquímico mostraron que la solución con $1 \%$ de $\mathrm{NaOH}$ y temperatura de $80^{\circ} \mathrm{C}$ fue la que presentó mayores ES en la superficie del PET. En relación a la separación del PVC por flotación, los resultados mostraron que el medio más eficiente de separación es el básico, llegando a alcanzar un $85 \%$ de separación de PVC en solución de tensoactivo de $0,25 \%$ y de $87 \%$ en solución de tensoactivo de $0,50 \%$.

Descriptores: PET, PVC, resíduo plástico, reciclaje, separación, tratamiento químico.

\begin{abstract}
One of plastics conventional but used by the society is the PET (tereftalato polythene), widely used in the production of bottles for carbonated drinks and mineral water. Another plastic that also is used for that application, but in smaller amount is the PVC, (vinyl polychloride). The polymer recycling is important and advantageous, but it also exists disadvantages like the great volume of generated remainder, exhaustion of the sanitary fillings, among others. Diverse techniques of separation of polymeric materials exist, being the most used, the separation by difference of densidades (gravity), due to that is one of the methods but easy and cheap to be applied, the great problem of that method is the separation of materials with similar densidades, as it is the case of PET and PVC, between 1.30-1.37 $\mathrm{g} / \mathrm{cm} 3$ disabling the separation of the same by that technique. The great problem is the contamination from PVC in the PET due to that it causes spots in the PET transparent as well its degradation. In this sense, the objective of that study is to determine the most efficient and economically viable methodology of separation from PVC in PET through flotation method. The first step was the treatment of the samples with alkaline solution of $\mathrm{NaOH}, 1.0$ and $4.0 \%$ in mass, at temperatures of $250 \mathrm{C}$ and $80 \mathrm{oC}$, and times of treatment of 15 and 30 minutes. For the determination of the surface energy was realised the measures of contact angle using a goniometer. In the flotation test, solutions of MIBC of 0.25 and $0.5 \%$ were used and different $\mathrm{pHs}$ (acid, basic and neutral). Results of the conditions of thermo-chemical treatment showed that the solution with $1 \%$ of $\mathrm{NaOH}$ and $80{ }^{\circ} \mathrm{C}$ was the one that presented higher surface energies in the surface of the PET. In relation to the separation of the PVC by flotation, results showed that the condition more efficient was the basic, getting to reach a $85 \%$ of separation of PVC in solution of tensoactivo of $0.25 \%$ and $87 \%$ in solution of tensoactivo of $0.50 \%$.
\end{abstract}

Keywords: PET, PVC, plastic waste, recycling, separation, chemical treatment 


\section{INTRODUCCIÓN}

Desde el descubrimiento del plástico, este material es cada vez más utilizado para diversas aplicaciones, desde embalajes simples para alimentos hasta materiales de construcción civil asi como en materiales compósitos de alto desempeño. varios tipos de materiales poliméricos se encuentran presentes en nuestro dia-a-dia, tales como el PP (polipropileno), PEAD (polietileno de alta densidad), PEBD (polietileno de baja densidad), PS (poliestireno), PVC (policloruro de vinil), PET (politereftalato de etileno) entre otros.

Las ventajas son grandes en la utilización de material plástico, de entre ellas estan: baja densidad, elevada protección y vida útil del producto almacenado. Por otro lado, asi como en cualquier situación, existen también algunas desventajas tales como: gran volumen de residuos, agotamiento de los rellenos sanitarios, entre los más importantes.

La creciente utilización de materiales poliméricos, genera una elevada cantidad de residuos y por consecuencia causa daños al medio ambiente que son inherentes a su descarte no racional, tornándose una gran preocupación mundial. La falta de hábitos de separación de los residuos post-consumo de la sociedad moderna y la falta de políticas que visen la reutilización y reciclaje de residuos poliméricos ha contribuido mucho para el agravamiento de este problema.

En los últimos años es posible identificar algunas actitudes referentes a mejorías en este sector, tales como estudios referentes a las técnicas innovadoras de reciclaje y el empeño de universidades e industrias en esa área [1,2]. Lo que ha motivado industrias y universidades a aplicar sus esfuerzos en la reciclaje, en grande parte es el costo /beneficio que esto puede traer como retorno. Así como la rentabilidad, la reciclaje también puede traer beneficios socio-económicos, tales como: mejorías en la calidad de vida de la población, generación de la renta, atenuación de problemas ambientales y economía de recursos naturales, entre otros [1]. El Brasil está entre los países que más reciclan materiales poliméricos en el mundo, solo pierde frente a los países europeos como Alemania, Bélgica, Suiza, Checoeslovaquia, Noruega, Suecia y Austria $[3,4]$.

El gran problema de los residuos poliméricos es el elevado tiempo que llevan para descomponerse permaneciendo por muchos años en la naturaleza, agravando los problemas ambientales y disminuyendo la vida útil de los rellenos sanitarios [5].

Normalmente las tres formas de reducir los resíduos plásticos son la reducción en la fuente, reutilización y reciclaje de diferentes formas, incluyendo el reciclaje energético. Esas iniciativas no acaban con el problema del residuo, pero contribuyen para la preservación del medio ambiente y evitando el agotamiento de los rellenos sanitarios [6].

Para amenizar esa situación esta siendo utilizada la práctica de los 3Rs: Reducir, Reutilizar y Reciclar, esas actitudes contribuyen para disminuir la utilización de la materia-prima directo de la fuente, así como reducir también la utilización de fuentes energéticas en la producción tanto de las resinas virgenes como en el reprocesamiento de los polímeros post-consumo [7].

EI PET está entre los polímeros más encontrados en el residuo sólido urbano (RSU). También los polímeros como PP y PVC presentan mayor producción en el Brasil, siendo utilizados como materia-prima de productos acabados con vida útil grande, normalmente estos son utilizados como materiales para construcción civil [8]. Al contrario del $\mathrm{PET}$, que es utilizado principalmente en embalajes tales como botellas de bebidas carbonatadas que presentan un tiempo de vida útil corta, generando así un gran volumen de ese residuo [4,9]. La gran variedad de residuos poliméricos y su tiempo de degradación relativamente grande hacen que ellos se tornen a los villanos ambientales [7]. Para solucionar este problema, lo ideal es utilizar un reciclaje sistemático de los polímeros, eso minimiza los impactos ambientales causados por esos resíduos. Lo ideal es que todas las ciudades tengan colecta selectiva y que la población adquiera el hábito de separar los residuos que pueden ser reciclados [10].

El reciclaje de materiales poliméricos post-consumo tiene como características y al mismo tiempo problemas, su contaminación (orgánica e inorgánica) y también la heterogeneidad de estos materiales debido a la gran variedad de materiales poliméricos descartados. Para que el producto reciclado tenga mejor aceptación en el mercado y menos problemas en el reprocesamiento, se hace necesario la remoción de las impurezas, así como la separación de los diversos tipos de polímeros. Cuanto más puro sea el residuo post-consumo, mejor será el valor del producto final [11]. 
Para agilizar la separación de los residuos poliméricos, diversas son las técnicas que pueden ser utilizadas para la separación y reciclaje de estos materiales, tales como fluorescencia de rayos $\mathrm{X}$, lecho fluidizado, separación por infrarrojo, flotación entre otros [12, 13]. Pero, el gran problema es que algunos de esos métodos poseen un costo elevado imposibilitando sus usos en empresas pequeñas. De entre los diferentes métodos mencionados el que posee menor costo es la separación por flotación, en la cual normalmente son utilizados tanques con agua, pero no todos los polímeros pueden ser separados por este método, generalmente eso ocurre en función de la diferencia de las densidades entre los polímeros [14]. Siendo el caso del PET y PVC, que poseen densidades semejantes, entre 1,33 y $1,37 \mathrm{~g} / \mathrm{cm}^{3}$, lo que hace que ambos se depositen en el fondo del tanque de flotación y por consecuencia dificulte la separación de estos dos polímeros [15].

La contaminación del PET por PVC causa problemas en el futuro reprocesamiento de este, pues la temperatura de procesamiento del PVC es inferior a la del PET, lo que causa varios problemas en el producto final (PET reciclado). De entre los problemas están la presencia de manchas oscuras en el PET reciclado, asi como la liberación de ácido clorhídrico al ambiente de trabajo y el desgaste de los equipos [16].

La dificultad de separación de residuos de PET y PVC es uno de los grandes problemas de la industria de reciclaje de PET, por lo tanto el objetivo de este trabajo es desarrollar las condiciones más eficientes de separación de PVC por el método de flotación después de un tratamiento termo-químico previo, para tornar la superfície de PET más hidrófila lo que llevará a su hundimiento mientras que el PVC más hidrofobito flotará.

\section{METODOLOGÍA}

\section{Materiales}

Para la realización de este estudio fueron utilizados botellas de PET post-consumo transparente proveniente de bebidas carbonatadas y de agua mineral. Las botellas fueron cortadas en pedazos pequeños de aproximadamente $8 \mathrm{~mm} \times 8 \mathrm{~mm}$ (flakes) con el auxilio de una tijera. El PVC utilizado fue proveniente de blister de remedio y fueron también cortados en forma de flakes.

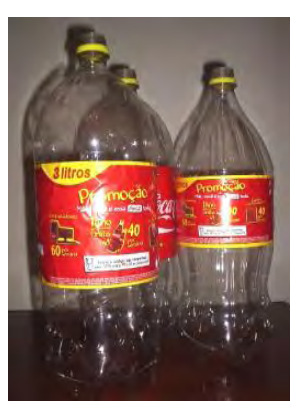

(a)

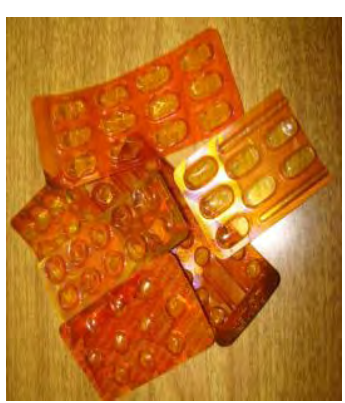

(b)
Figura 1: Muestras de resíduo plástico post-consumo (a) botellas de PET y (b) blister de PVC.

\section{Tratamiento termo-químico}

Las muestras de PET y PVC fueron sometidas a 8 condiciones de tratamiento termo-químico (soluciones de $\mathrm{NaOH}$ a diferentes concentraciones, temperatura y tiempo) como mostrado en la Tabla 1 , para remoción de las impurezas contenidas en la superficie de los flakes.

Tabla 1: Condiciones de tratamiento termo-químico de los flakes de PET/PVC.

\begin{tabular}{|c|c|c|c|c|}
\hline Muestra & $\begin{array}{c}\mathbf{N a O H} \\
(\% \mathbf{~ m} / \mathbf{m})\end{array}$ & $\begin{array}{c}\text { Tiempo } \\
\text { del baño } \\
(\mathbf{m i n})\end{array}$ & $\begin{array}{c}\text { Temperatura } \\
\text { del baño } \\
\left({ }^{\circ} \mathbf{C}\right)\end{array}$ & $\begin{array}{c}\text { Temperatura } \\
\text { de secado } \\
\left({ }^{\circ} \mathbf{C}\right)\end{array}$ \\
\hline A1 & 1,0 & 15 & 25 & 80 \\
\hline A2 & 1,0 & 30 & 25 & 80 \\
\hline A3 & 1,0 & 15 & 80 & 80 \\
\hline A4 & 1,0 & 30 & 80 & 80 \\
\hline A5 & 4,0 & 15 & 25 & 80 \\
\hline A6 & 4,0 & 30 & 25 & 80 \\
\hline A7 & 4,0 & 15 & 80 & 80 \\
\hline A8 & 4,0 & 30 & 80 & 80 \\
\hline
\end{tabular}

Para la realización del tratamiento químico fueron sometidas cantidades conocidas de flakes de PET y PVC para facilitar la visualización, en la siguiente etapa de separación por flotación.

Después del tratamiento químico las muestras de PET/PVC fueron enjuagadas en agua con $\mathrm{pH} 7,0$ y en la secuencia sometidas a los ensayos de flotación, con excepción de las muestras utilizadas para los ensayos de ángulo de contacto y energía superficial, las cuales después del enjuague fueron secadas por un período de 3 horas en estufa.

Para los ensayos de flotación fueron utilizados soluciones de MIBC en las concentraciones de 0,25 e $0,50 \%$ con pH ácido, neutro y básico. Los flakes de PET e PVC fueron colocados en estas soluciones y con flujo de aire (para generación de burbujas). 


\section{Caracterización}

La determinación de la eficiencia de separación de PVC fue a través de la cuantificación de los flakes de $P V C$ que flotaron y que fue realizada visualmente, ya que las cantidades de flakes de PET y PVC en cada muestra eran conocidas.

El otro grupo de muestras de PET e PVC tratadas y secas fueron caracterizadas a través del análisis del ángulo de contacto y determinación de la energia superficial, para lo cual fue utilizado agua y diyodometano $\left(\mathrm{CH}_{2} \mathrm{I}_{2}\right)$. Las medidas fueron hechas con el goniómetro Ramé-Hart modelo 100-00, conforme la norma ASTM D 724-89. Los valores del ángulo de contacto fueron utilizados para calcular la energía de superficie conforme la ecuación (a):

$$
\gamma_{t}=\gamma^{p}+\gamma^{d} \ldots
$$

Donde $\gamma_{t}$ es la energía total de superficie $\left(\mathrm{T}_{\mathrm{t}}\right) ; \gamma^{p}\left(\mathrm{~T}_{\mathrm{p}}\right)$ y $\gamma^{d}\left(T_{d}\right)$ son las componentes polar y disperso de la energía de superficie respectivamente [17].

\section{RESULTADOS Y DISCUSIÓN}

\section{Ângulo de contacto}

Para la evaluación de los cambios físicos en la superficie del PET y PVC después del tratamiento químico, en la Figura 2 son mostradas las imágenes de gotas de agua en la superficie de las muestras PET y PVC tratadas con solución de $\mathrm{NaOH} 1 \%$ y temperatura de $25^{\circ} \mathrm{C}$.

Se observa en las Figuras 2-a y 2-c que las muestras de PVC son más hidrofóbicas que las de PET debido al menor espallamiento de la gota de agua sobre la superfície de la muestra cuando comparado con el formato de las gotas en la superfície de PET (Figuras 2-b y 2-d). Por lo tanto, los ângulos de contacto de agua con la superfície de PVC son mayores que las del PET.

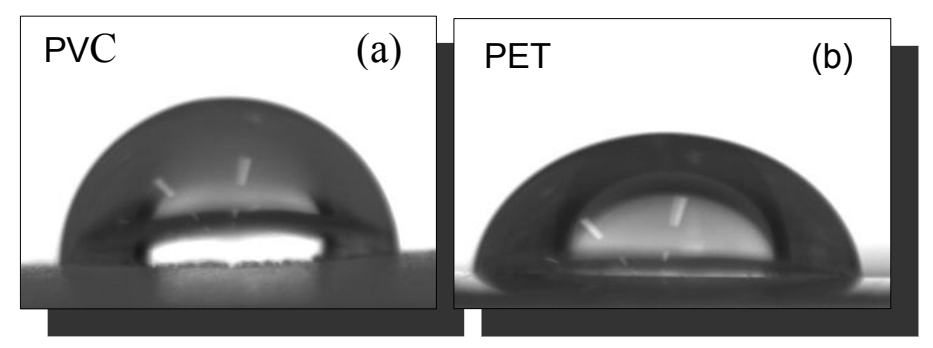

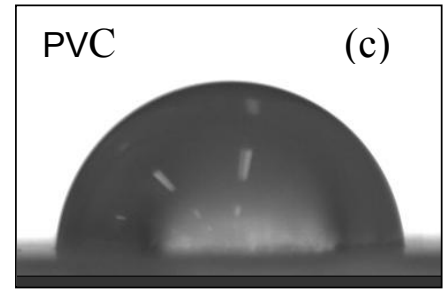

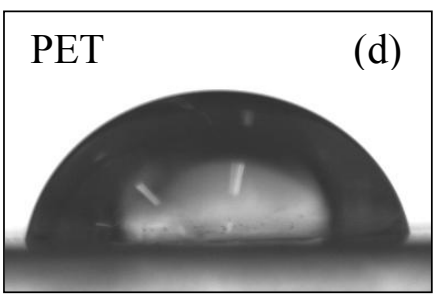

Figura 2: Gota de agua sobre substrato polimérico tratados con solución de $\mathrm{NaOH} 1 \%$, a $25^{\circ} \mathrm{C}$ y tiempo de baño de: (a) PVC, $15 \mathrm{~min}$; (b) PET $15 \mathrm{~min}$; (c) PVC, 30 min; $y$ (d) PET, 30min.

En la Figura 3 son mostradas las imágenes de las gotas de agua en los substratos de PVC y PET después del tratamiento con solución de $\mathrm{NaOH} 4 \%$, por 30 minutos y con una mayor temperatura de baño $\left(80^{\circ} \mathrm{C}\right)$, donde también es observado que el formato de la gota más esparcida es en la superfície sólida correspondiente al PET.
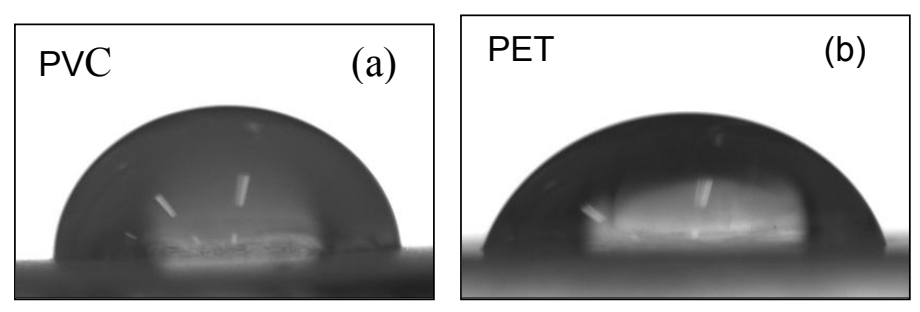

Figura 3: Gota de agua sobre substrato polimérico tratados con solución de $\mathrm{NaOH} 4 \%$, a $80^{\circ} \mathrm{C}$ y tiempo de baño de: (a) PVC, $30 \mathrm{~min}$; (b) PET $30 \mathrm{~min}$.

Comparando las condiciones de tratamiento más agresivo, en las Figuras 2-c y 2-d con las Figuras 3-a y 3-b, obsérvase que el aumento de la concentración de $\mathrm{NaOH}$ de $1 \%$ para $4 \%$ y de la temperatura de $25^{\circ} \mathrm{C}$ para $80^{\circ} \mathrm{C}$ influenció en la disminución del ángulo de contacto de agua con la superfície polimérica de ambos, pues es visualizado un mayor esparcimiento de la gota. Resultado interesante que puede estar indicando variaciones en la energia superficial de la superfície del substrato polímérico.

En la Tabla 2 son mostrados los resultados de ángulos de contacto formados entre los líquidos usados (agua y $\mathrm{CH}_{2} \mathrm{l}_{2}$ ) y las superfícies de los substratos tratados, donde todos los ângulos formados con agua fueron superiores que con el $\mathrm{CH}_{2} \mathrm{I}_{2}$. Se observa también que los ángulos formados com agua con la superfície de PET fueron generalmente inferiores al del PVC, resultados que confirman las imágenes de las gotas de agua más esparcidas sobre la superfície de PET de la Figuras 2-b, 2-d e 3-b. 
Tabla 2: Resultados de los ángulos de contacto de los substratos de PET y PVC.

\begin{tabular}{|l|c|c|c|c|}
\hline & \multicolumn{2}{|c|}{ PET } & \multicolumn{2}{c|}{ PVC } \\
\hline & $\theta_{(\mathrm{H} 2 \mathrm{O})}$ & $\theta_{(\mathrm{CH} 212)}$ & $\theta_{(\mathrm{H} 2 \mathrm{O})}$ & $\theta_{(\mathrm{CH} 212)}$ \\
\hline A1 & 80,9 & 41,2 & 88,0 & 44,6 \\
\hline A2 & 78,2 & 38,8 & 80,1 & 45,9 \\
\hline A3 & 77,1 & 37,9 & 80,3 & 45,9 \\
\hline A4 & 74,1 & 37,7 & 81,7 & 52,2 \\
\hline A5 & 77,1 & 42,4 & 86,0 & 58,4 \\
\hline A6 & 79,7 & 39,8 & 85,0 & 65,8 \\
\hline A7 & 72,4 & 43,1 & 93,0 & 60,8 \\
\hline A8 & 80,8 & 40,5 & 87,9 & 60,3 \\
\hline
\end{tabular}

\section{Energia de superfície}

Los resultados del ensayo de ángulo de contacto fueron utilizados para la determinación de la energía de superficie de los substratos evaluados conforme están presentados en los resultados en la Figura 4.

Se observa que todas las muestras de PET tratadas en las 8 condiciones (Tabla 1) presentaron una energía superficial superior al del PVC, confirmando los resultados de ángulo de contato, donde la superficie de PVC es más hidrofóbica que del PET.

También se nota que el aumento de la concentración de la solución de $\mathrm{NaOH}$ de 1 para $4 \%$ influenció en el aumento de la diferencia de la energia superficial entre ambos substratos (PET e PVC), aunque estos valores sufrieron una disminución ( $A 6, A 7$ y $A 8)$ cuando comparados con las condiciones (A3 y A4), resultados que pueden ser debidos a la degradación que pueden haber sufrido las superficies de ambos sustratos.

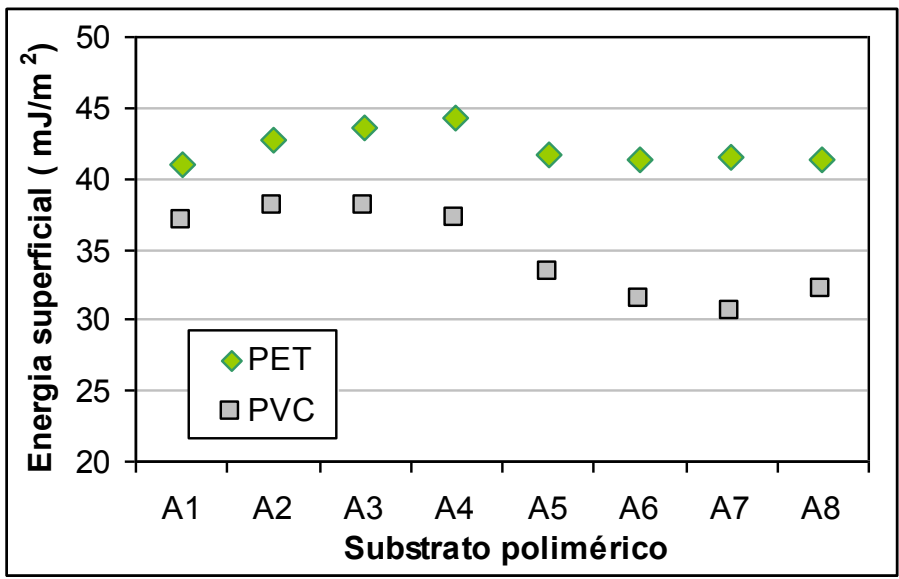

Figura 4: Energía superfical de las muestras de PET y PVC en las 8 condiciones de tratamiento termoquímico.

\section{Separación por flotación}

La comprobacion final de que hubo variación de la energia de superfície de los flakes de PET y PVC fue determinada por los resultados obtenidos en el ensayo de flotación, donde se confirman las informaciones obtenidas en los ensayos de ángulo de contacto y energía de superficie, los cuales muestran que el PET después del tratamiento químico se torna menos hidrofóbico que el PVC, hecho que propicia la flotación del PVC y el PET permanezca en el fondo del recipiente como es mostrado en la Figura 5

En el proceso de flotación, la especie más hidrofóbica fluctúa (PVC), pues esta presenta menor energia superficial lo que favorece la separación por flotación. Ya la especie menos hidrofóbica (hidrofílica) se deposita en el fondo del recipiente por presentar mayor energia de superfície.
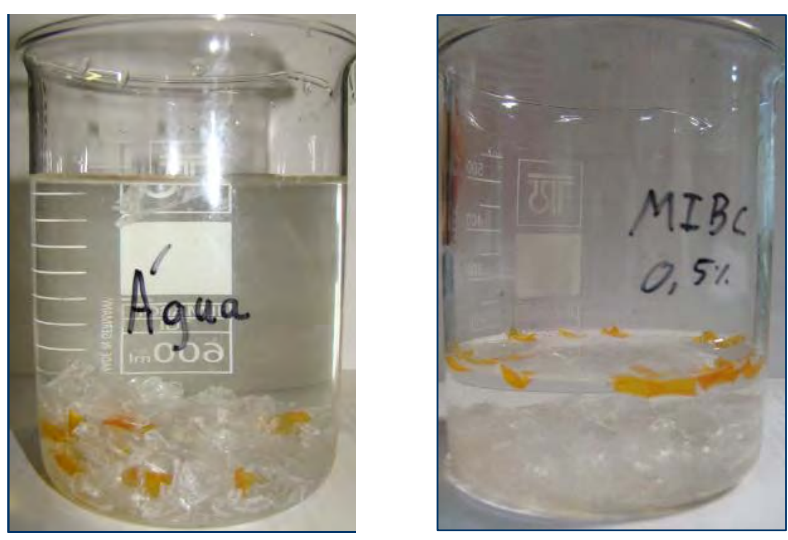

Figura 5: Flakes de PET y PVC de la condición A8 despues del ensayo de flotación en (a) agua y (b) solución de MIBC, 0,5\%.

Para la determinación de la eficiencia de separación de PVC en el PET, fueron testados en dos concentraciones del tensoactivo MIBC y variando el $\mathrm{pH}$ de la solución.

Los resultados de esta evaluación son mostrados en la Figura 6 , donde se observa que las mayores eficiencias de separación de PVC por flotación es en medio básico tanto para las concentraciones de MIBC de 0,25 como 0,5\%.

La mayor eficiencia de separación fue alcanzada con la muestra $\mathrm{A} 8$ que fue tratada en solución de $\mathrm{NaOH}$ $4 \%$ a $80^{\circ} \mathrm{C}$ por 30 min y el ensayo de flotación realizada en solución básica con concentración de 0,50\% de MIBC como muestra la Figura 6-b. 


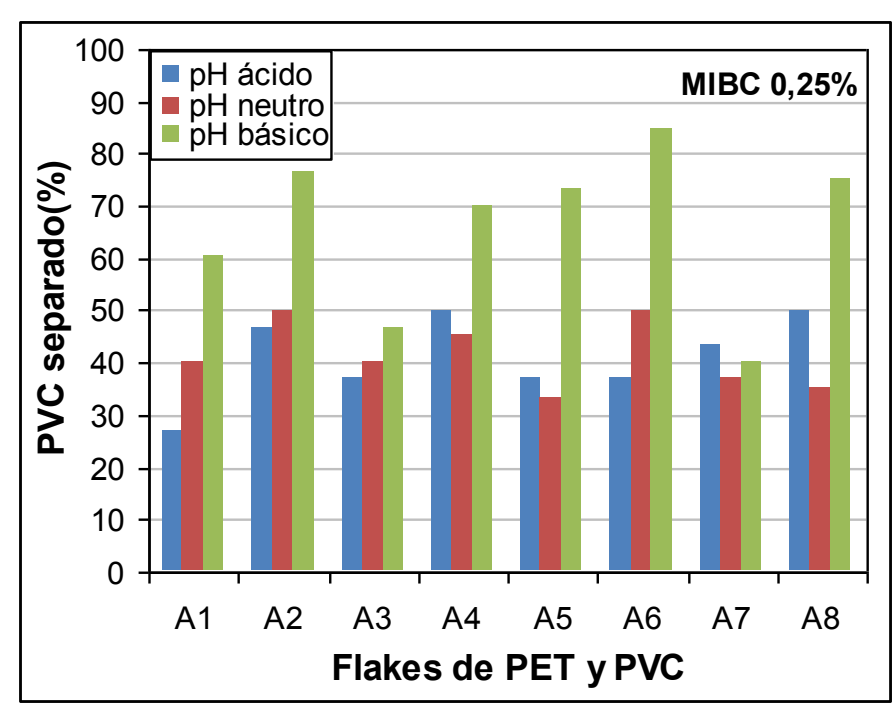

(a)

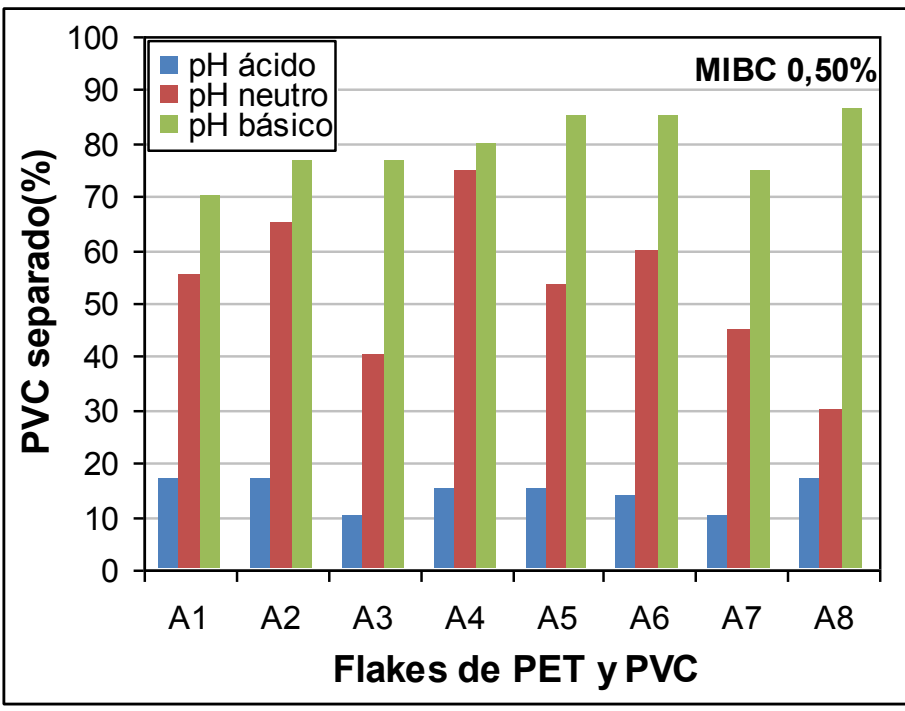

(b)

Figura 6: Eficiencia de separación de PVC por flotación en funcion de $\mathrm{pH}$ de la solución de MIBC de (a) $0,25 \%$ y (b) $0,5 \%$.

Aunque la solución de tensoactivo de $0,5 \%$ de MIBC obtuvo mejores resultados que el $0,25 \%$, no podemos dejar de considerar que en la solución de $0,25 \%$ de $\mathrm{pH}$ básico también fueron obtenidos buenos resultados de flotación, como es el caso de las muestras A2 (tratada en la solución de $\mathrm{NaOH} 1 \%$ a $24^{\circ} \mathrm{C}$ por $30 \mathrm{~min}$ ) en la cual fluctuó $77 \%$ del PVC y así como en la muestra $A 6$, que se obtuvo una $85 \%$ de eficiencia de separación de PVC.

Pero como el objetivo de la investigación es desarrollar un método fácil y económicamente viable para ser utilizado en empresas pequeñas de reciclaje de PET, se puede concluir que la mejor condición es A2 en médio básico y con concentración de tensoactivo de $0,25 \%$ de MIBC en la cual son utilizados menores cantidades de reactantes y menos energía pues el tratamiento es realizado a temperatura ambiente $\left(25^{\circ} \mathrm{C}\right)$.

\section{CONCLUSIONES}

De los resultados obtenidos en este trabajo se puede concluir que el tratamiento termo-químico realizado con solución de $1 \%$ de $\mathrm{NaOH}$ y aumento de la temperatura de $25^{\circ} \mathrm{C}$ para $80^{\circ} \mathrm{C}$ influenció grandemente en los cambios de energia de superfície de los substratos poliméricos evaluados, tornando las superfícies menos hidrofóbicas (PET) favoreciendo su separación con el PVC por flotación. Siendo confirmados por los resultados de ángulo de contacto y energía superficial, donde todas las muestras de PVC presentan mayores ángulos de contacto y por lo tanto menores energías de superficie que las muestras de PET.

De los resultados obtenidos por flotación se observa que el medio más eficiente de separación es el básico, llegando a alcanzar un $85 \%$ de separación de PVC en solución de tensoactivo de $0,25 \%$ y de $87 \%$ en solución de tensoactivo de $0,50 \%$.

Como el foco del estudio es desarrollar un método de separación para ser utilizado en micro y pequeñas empresas de reciclaje de PET, las cuales no disponen de muchos recursos financieros, se concluye que la mejor condición de tratamiento químico (menos agresivo evitando la degradación del PET), es la de la muestra A2 (solución $\mathrm{NaOH}$ $1 \%$, a $25^{\circ} \mathrm{C}$, por 30 minutos), y la solución de separación por flotación el MIBC $0,25 \%$ en $\mathrm{pH}$ básico; presentando $77 \%$ de eficiencia de separación de PVC en el PET.

\section{AGRADECIMIENTOS}

Agradecemos al Laboratorio LACER-UFRGS por permitir el uso del goniómetro, sin la cual no seria posible la realización de los ensayos

\section{REFERENCIAS}

[1] F. S. Forlin, J.A.F. Farias, Polímeros: Ciência e Tecnologia, vol 12, n 1, 2002, 1-10.

[2] G. GÜÇLÜ, T. YALÇINYUVA, S. ÖZGÜMÜS, M. ORBAY, Thermochimica Acta, v.404, 2003, 193-205.

[3] W. Romão, M. A. S. Spinacé, M. A. De Paoli, Polímeros: Ciência e Tecnologia, vol 19, n 2, 2009, 121-132. 
[4] http/www.plastivida.org.br, acessado em dezembro de 2010.

[5] S. L. F. Gonçalves-Dias, A. S. S. Teodósio.Produção, v. 16, n. 3, 2006, p. 429441.

[6] A. S. F. Santos, J. A. M. Agneli, S. Manrich, Polímeros: Ciência e Tecnologia, vol. 14, $n^{\circ} 5$, p. 307-312, 2004

[7] S. L. F. Gonçalves-Dias, Gestão e Produção, v.13, n.3, 2006, p.463-474.

[8] S. D. Mancini, I. G. Matos, R. F. Almeida, Polímeros: Ciência e Tecnologia, vol 14, n 2, 2004, 69-73.

[9] http/www.abiplast.org.br, acessado em dezembro de 2010.

[10] L. Coltro, A. E. P. Buratin. Polímeros: Ciência e Tecnologia, vol 14, n 3, 2004, p. 206-211.

[11] http/www.institutodopvc.org.br, acessado em dezembro de 2010.

[12] M.T. CARVALHO, C. FERREIRA, A. PORTELA, J.T. SANTOS, Waste Management, v.29, 2009, 1138-1143.

[13] F. Awaja, D. Pavel, European Polymer Journal, vol 41, 2005, p 1453-1477

[14] H. Shen, R.J. Pugh, E. Forssberg, Colloids and Surfaces A: Physicochemical and Engineering Aspects, vol 196, 2002, p 63-70.

[15] P. C. Dartora, E. N. Bubicz, A. K. R. Oliveira, R. M. C. Santana Proceedings of the Polymer Processing Society 26th Annual Meeting PPS-26 July 4-8, 2010 Banff (Canada)

[16] J. DRELICH, T. PAYNE, J. H. KIM, J. D. MILLER. Polymer Engineering and Science, v.38, n.9, 1998, p.1378-1386.

[17] R. M. C SANTANA, S. MANRICH, Jornal Apllied Polymer Science, v.114, 2009, 34203427.

E-mail: ruth.santana@ufrgs.br 